\title{
Laboreal
}

Volume $3 \mathrm{~N}^{\circ} 1$ | 2007

Varia

\section{Sufrimiento físico, psíquico y moral en el sector del calzado en Portugal : aportaciones para una psicodinámica del trabajo}

Sofrimento físico, psíquico e moral no sector do calçado em Portugal :

contributos para uma psicodinâmica do trabalho

Souffrance physique, psychique et morale dans le secteur de la chaussure au

Portugal : Contribution pour une psychodynamique du travail

Physical, psychological and moral suffering in the foot-wear industry in

Portugal : contributions to work psychodynamics

\section{Clara de Araújo}

\section{(2) OpenEdition}

\section{Journals}

Edición electrónica

URL: http://journals.openedition.org/laboreal/12917

DOI: $10.4000 /$ laboreal. 12917

ISSN: 1646-5237

Editor

Universidade do Porto

Referencia electrónica

Clara de Araújo, « Sufrimiento físico, psíquico y moral en el sector del calzado en Portugal

aportaciones para una psicodinámica del trabajo », Laboreal [En línea], Volume $3 N^{0} 1$ | 2007, Publicado el 01 julio 2007, consultado el 24 septiembre 2020. URL : http://journals.openedition.org/laboreal/ 12917 ; DOI : https://doi.org/10.4000/laboreal.12917

Este documento fue generado automáticamente el 24 septiembre 2020

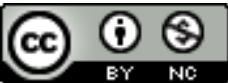

Laboreal está licenciado com uma Licença Creative Commons - Atribuição-NãoComercial 4.0 Internacional. 


\section{Sufrimiento físico, psíquico y moral en el sector del calzado en Portugal : aportaciones para una psicodinámica del trabajo}

Sofrimento físico, psíquico e moral no sector do calçado em Portugal :

contributos para uma psicodinâmica do trabalho

Souffrance physique, psychique et morale dans le secteur de la chaussure au

Portugal : Contribution pour une psychodynamique du travail

Physical, psychological and moral suffering in the foot-wear industry in

Portugal : contributions to work psychodynamics

Clara de Araújo

REFERENCIA

Araújo, C. (2003). Sofrimento Físico, Psíquico e Moral no sector do calçado em Portugal. Contributos para uma Psicodinâmica do Trabalho. Tese de Doutoramento. Faculdade de Psicologia e Ciências de Educação da Universidade do Porto, Porto.

\section{NOTA DEL EDITOR}

Manuscrito recibido en : abril/2007

Aceptado tras peritage en : julio/2007 


\section{Contextualización de la problemática}

1 El punto de partida de este trabajo transcurre de reflexiones teórico-metodológicas, alrededor de la función del trabajo en la vida de las personas y en particular de su salud y remite hacia la cuestión: ¿De qué forma el trabajo puede generar sufrimiento contribuyendo para el desequilibrio de la salud en algunos trabajadores? y, ¿Cómo es que en otras situaciones de trabajo acaba por constituirse factor estructurador, pudiendo observarse que es entonces, por el trabajo que las personas acaban por defender, de forma holistica su equilibrio psicofisiológico y social, construyendo su salud? En este sentido, definimos como objetivos de este estudio el análisis del sufrimiento en y por el trabajo en sus dimensiones física, psíquica y moral y, la comprensión del dominio del sufrimiento y de las estrategias defensivas construidas por los sujetos para evitar oscilar en la descompensación psicopatológica.

2 Consideramos la salud en la óptica de la normalidad según Dejours (1995), es decir, partimos de la suposición de que la salud no es un don natural y no existe salud perfecta. La salud se construye, en el sentido de la obtención de un equilibrio precario pero aceptable donde el sufrimiento no está ausente pero es compensado.

3 A la luz de la concepción de las relaciones del hombre con el trabajo, se justifica, un triángulo fundamental, el de la dinámica de la identidad de F. Sigaut constituido por tres extremos : el del YO - el del REAL - el del OTRO, que toma en psicodinámica del trabajo una forma específica - la del triángulo de la psicodinámica del trabajo. En este, el YO es un sujeto de sufrimiento, el REAL es indexado al trabajo y el OTRO substituido por el reconocimiento (Dejours, 2000). Este triángulo, modeliza el principio según el cual las relaciones entre un sujeto (yo) y un grupo (otro) no se reducen a simples relaciones de poder o de reconocimiento, pero son siempre profundamente enraizadas en un acto, en una actividad, en una conducta individual sobre el "REAL". De ahí que la realización del YO, pasa necesariamente por una mediatização, en la relación a lo REAL que constituye el trabajo.

4 Así, la negación y/o el no reconocimiento de la realidad del trabajo de una persona puede ser fuente de sufrimiento mental.

5 Pero, en contrapartida, el reconocimiento en el trabajo es un elemento determinante de la realización de la persona y contribuye para la construcción de su identidad.

\section{Tres niveles de análisis}

6 De esta forma consideramos que cada uno de los tres estudios que desarrollamos se sitúa de forma privilegiada en uno de los tres extremos do triangulo de Sigaut, aunque nuestro análisis se haya enriquecido progresivamente aproximándose a poco y poco de la riqueza de las aportaciones de la psicodinámica del trabajo.

7 Nuestro primer estudio - en realidad conducido en una fase anterior a nuestra inmersión en la obra de Dejours - privilegia el YO - quiere decir, el sujeto que trabaja. Las suposiciones teórico-metodológicos del estudio fueron los del planteamiento tradicional al estrés ocupacional y a su influencia en la salud mental del trabajador. Se comparó un grupo de individuos desempleados con otro de individuos empleados, privilegiándose un conocimiento en extensión, más que un conocimiento en comprensión, habiéndose utilizado la escala S.C.L. - 90-E para medir la salud mental de 
los individuos y una escala de Fuentes de Estrés en el Trabajo para registrar las fuentes de estrés que pudiesen descompensar al individuo. Todavía, el análisis del sufrimiento en el trabajo también es influenciado por las condiciones de trabajo.

8 Es así que el segundo estudio pasa a privilegiar el extremo de lo REAL - es decir, el lugar del trabajo, habiendo la opción metodológica sido la de un estudio de caso en una empresa de calzado orientado hacia el análisis de la actividad del trabajo, permitiendo construir un mejor conocimiento de la actividad de los trabajadores del sector del calzado.

El tercer estudio privilegió el extremo de los OTROS, es decir, los pares, la jerarquía y los subordinados, en la suposición de que la relación con el trabajo no es apenas técnica, cognitiva o física, pero sometida a un contexto intersubjetivo que es central en el análisis del sufrimiento. En este cuadro los otros son los trabajadores del calzado que se reunieron voluntariamente en grupo con los investigadores en sesiones de análisis colectiva de trabajo (Ferreira, 1993; 2001), habiéndose constituido un espacio de palabra donde emergieron conocimientos hasta ahí ocultados sobre el trabajo real.

\section{Contexto de la investigación}

El contexto de la investigación se desarrolló en el sector de los trabajadores del calzado. En el primer estudio que tuvo como paño de fondo la evaluación de la salud mental, se crearon dos grupos de trabajadores, uno de empleados y otro de desempleados. Los primeros pertenecían a varias empresas del sector secundario, pero una buena parte de la muestra provenía de una empresa de calzado.

11 El hecho de que la parte de la muestra de la primera pesquisa perteneciese a una empresa del sector del calzado donde teníamos la posibilidad de conducir otro estudio, fue entonces determinante para el privilegio atribuido al contexto específico de los trabajadores, por lo que el segundo estudio se realizó exclusivamente en una empresa de calzado.

El tercer estudio, teniendo en cuenta la opción metodológica por el Análisis Colectivo del Trabajo, fue conducido con la colaboración y participación de la organización sindical del correspondiente sector habiéndose constituido dos grupos de trabajadores del calzado, en locales de inserción geográfica diferentes de la región Norte del País, habiéndose respetado los principios del voluntariado y del anonimato.

\section{Resultados de los estudios}

Al comprobar en el primer estudio que la salud mental de los individuos desempleados no se presentaba peor que la de los empleados estábamos ante un resultado importante para la evolución de nuestra reflexión en el plan metodológico orientando nuestra mirada hacia la población empleada.

Este primer estudio fue muy útil por habernos confrontado con un paradigma de investigación epidemiológica, impidiendo de analizar el proceso de interacción entre el individuo y la situación de trabajo.

Es así, que surge la necesidad de orientar la investigación en el análisis del sufrimiento para una aproximación a lo real del trabajo privilegiándose principios metodológicos de 
la ergonomía, habiéndose encontrado en el caso analizado perturbaciones infrapatológicas que confirman la hipótesis de existencia de condiciones de trabajo favorecedoras del aparecimiento y desarrollo de perturbaciones músculo-esqueléticas.

El último estudio cuya opción fue el planteamiento por el Análisis Colectivo del Trabajo permitió verificar que el sufrimiento físico referido está lleno de características que lo asemejan a un sufrimiento psíquico y moral y que se enraíza en las relaciones establecidas con la jerarquía por la presencia de miedo y de la presión que la misma ejerce. Sin embargo la actividad en sus relaciones con la jerarquía sigue siendo un peso de lo que en la organización es generador de sufrimiento y que los trabajadores realzaron como fuente de sufrimiento que se distingue de los resultados del segundo estudio. El reconocimiento limitado relativamente a la riqueza del saber por experiencia de los trabajadores, es una de las quejas expresadas por los mismos de que no siempre los encargados y jefes reconocen el valor del trabajo del operador.

Las formas encontradas por los trabajadores para superar el sufrimiento, es decir, las estrategias de defensa individual y colectivo eran guiadas por un fuerte individualismo, estimuladas por el peso de la tarea. La principal defensa psicológica es la negación. Para encontrar forma de convivir con el sufrimiento y con la situación de trabajo que lo provocó, los trabajadores niegan las situaciones peligrosas coercitivas o penosas y evitan la percepción del propio miedo. Para expulsar el miedo y permitir la convivencia con los riesgos inherentes al ambiente del trabajo ridicularizan verbalmente las fuentes de riesgo. En las relaciones con la jerarquía los trabajadores hacen humor con las situaciones o procuran el aislamiento de que es ejemplo "hacer que no oye".

\section{El reconocimiento del sufrimiento en la prosecución de la salud de los trabajadores}

18 En esta tesis pretendemos reflexionar la cuestión del sufrimiento en el trabajo, es decir, la salud y la forma como es regulada. Sin embargo, la poca valorización del trabajo actual y la fragilización del movimiento sindical constituyen factores que han contribuido para la ausencia de un reconocimiento social del sufrimiento de los trabajadores, teniendo sin embargo los trabajadores en las sesiones de ACT referido que les gustaría dar a conocer al exterior lo que ocurre en el trabajo. Hay por lo tanto necesidad de reconocer la salud en su concepción global, en sus dimensiones física, psíquica y moral, lo que exige superar la concepción dominante de que riesgo para la salud se reduce a un acontecimiento que se traduce en un daño físico y visible y reconocer el desgaste en el trabajo con resultado de un proceso largo y difícil. Sin embargo la evolución del trabajo condujo a nuevas formas de organización y a nuevos riesgos de los cuales señalamos las perturbaciones músculo-esqueléticas que en nuestro estudio se evidenciaron potenciales y que siendo de instalación insidiosa, el recorrido va siendo mediado por el sufrimiento de los trabajadores.

Por fin, este trabajo acabó por conducirnos a superar el campo de la psicodinámica del trabajo, sobretodo en lo que Dejours define como de contenido, de significado y de las formas de sufrimiento, sobretodo porque sitúa la investigación al nivel de lo infrapatológico denominándolo de - normalidad. Consideramos con Schwartz (1997), que toda actividad de trabajo es siempre "una dramática del uso de si", una negociación de cariz problemático entre el uso de si por si y el uso de si por los otros, en que los operadores realizan su actividad en función de arbitrajes constantes asociadas a la 
instantaneidad y heterogeneidad de los valores de la gestión "en si" y "de si". Así, aunque la psicodinámica del trabajo nos haya permitido concluir la importancia de los valores en la integración de las relaciones que atraviesan el cotidiano de la actividad de trabajo, Schwartz (1997) demuestra la conexión entre la dimensión ética y la dinámica social del reconocimiento, habiéndose verificado eso en nuestro último estudio.

Así, el recorrido de descubrimiento que hicimos parece habernos conducido a superar el paradigma de la normalidad que defiende Dejours (1995) para considerar el paradigma de la historia de vida (Davezies, 1998). De hecho, para todos, el trabajo constituye una experiencia de placer y de sufrimiento que estructura la existencia - es lo vivido de los trabajadores que estructura su capacidad de recuperar cuyo objetivo es : sentirse en forma, tener dominio sobre sus actos y tener una vida que tiene sentido. En la perspectiva de que el ser humano no procura adaptarse pero crecer.

\section{BIBLIOGRAFÍA}

Davezies, P. (1998). De la récupération psychique. Santé et Travail, 25, 35-37.

Dejours, C. (1995). Comment Formuler une Problématique de la Santé en ergonomie et en Médecine du Travail ? Le Travail Humain, 58, (1), 116.

Dejours, C. (2000). Travail, usure mentale. Paris : Bayard.

Ferreira, L.L. (1993). Análise Colectiva do Trabalho. Revista Brasileira de Saúde Ocupacional, 21, (78), 7-19.

Ferreira, L.L. (2001). A Análise Colectiva do Trabalho e seus usos em pesquisas e formação sindicais. Comunicação apresentada no Séminaire International Analyses du Travail et Formation no CNAM (mimeografado) 7pag.

Schwartz Y. (1997). Reconnaissances du Travail : Pour une approche ergologique. Paris : Presses Universitaires de France (PUF).

\section{AUTOR}

\section{CLARA DE ARAÚJO}

Faculdade de Motricidade Humana Estrada da Costa, Dafundo, 1495 - 688 Lisboa

ClaraAraujo@esenfvc.pt 